\title{
Hadron Spectroscopy at Jefferson Laboratory
}

\author{
Dennis P. Weygand \\ Thomas Jefferson National Accelerator Facility \\ Newport News, Virginia
}

\begin{abstract}
Recent results on hadron spectroscopy from Jefferson Laboratory's CEBAF Large Acceptance Spectrometer (CLAS) are presented. In particular we present results from the baryon resonance program for both electro- and photo- production. Also, we present very preliminary results on meson spectroscopy in $\gamma p$ interactions, and new results on the observation of the exotic baryon, the $\Theta^{+}$.
\end{abstract}

\section{INTRODUCTION}

The Continuous Electron Beam Accelerator Facility (CEBAF) has been been operational since 1995 . The accelerator provides an electron beam up to $6 \mathrm{GeV}$ which is separated and sent to three halls simultaneously. Halls $\mathrm{A}$ and $\mathrm{C}$ both are low acceptance double arm spectrometers restricted to an electron beam, while Hall $\mathrm{B}$, which contains the CEBAF Large Acceptance Spectrometer (CLAS) [1], also has a bremsstrahlung induced photon tagging system [2] to permit both electron and photon beams. Hall A consists of two identical high resolution spectrometers with maximum momentum of $4 \mathrm{GeV} / \mathrm{c}$, while Hall C consists of two symmetric focusing spectrometers, one with acceptance for high momentum particles, and the other for the detection of decay products. A schematic of the accelerator facility is shown in Fig. 1. Almost all of the hadron spectroscopy studies at CEBAF are performed at the CLAS facility, which began physics operations in 1997. CLAS is based on a six coil toroidal magnet which provides a primarily azimuthal field distribution, and is described in detail in Reference 1. Charged particle trajectory analysis from drift chambers [3] provides momentum resolution of $0.5 \%$ in the forward direction. A time-of-flight scintillator detector, and electromagnetic lead-scintillator sandwich calorimeters provide good particle identification, and a Cerenkov counter provides electron identification. Large acceptance is required to provide high reconstruction efficiency for multi-particle final states typical of hadronic reactions containing excited meson and baryon states. The luminosity of experiments using the tagged photon facility is limited to about $10^{7}$ photons/second due to accidental coincidences with the tagger, while electron beam luminosities are limited by rates in drift chambers. A schematic of the detector is shown in Fig 2. 


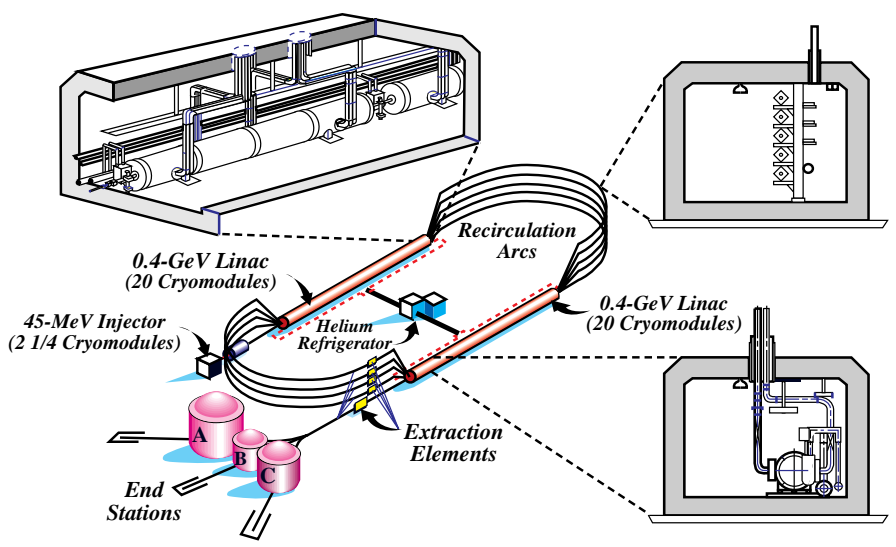

FIGURE 1. The CEBAF accelerator site.

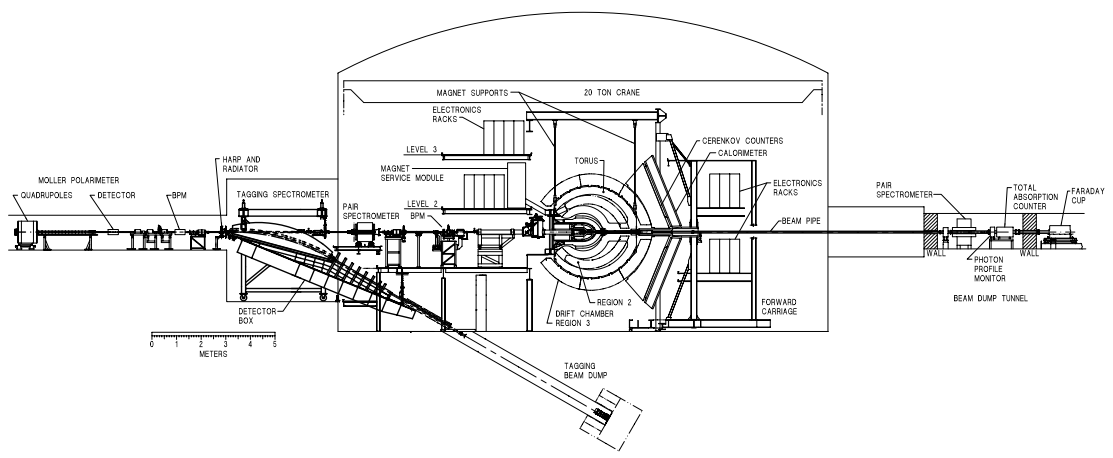

FIGURE 2. Side view schematic of the CLAS detector.

\section{THE FINAL STATE $\pi^{+} \pi^{-} p$}

Electromagnetic excitations of nucleon resonances, which are sensitive to the spin and spatial structure of the transition, give information on the properties of baryon structure. In the mass region above $1.6 \mathrm{GeV} / \mathrm{c}^{2}$ there are many overlapping states, many of which are not well known. In addition, many of these states may decouple from single meson channels, and thus decay mainly to multi-pion final states, such as $\Delta \pi$ and $N \rho$, and therefore $N \pi \pi$. SU(6) X O(3) symmetry predicts many more states than have been observed experimentally: QCD mixing effects could decouple these states from the $N \pi$ channel, while strongly coupling them to the $N \pi \pi$ channel. CLAS has taken data on the reactions $e p \rightarrow e^{\prime} p \pi^{+} \pi^{-}$and $\gamma p \rightarrow p \pi^{+} \pi^{-}$. In the electroproduction reaction, the data was divided into three bins of $Q^{2}$ : $0.5-0.8(\mathrm{GeV} / \mathrm{c})^{2}, 0.8-1.1(\mathrm{GeV} / \mathrm{c})^{2}$, and $(1.1-1.5)$ $(\mathrm{GeV} / \mathrm{c})^{2}$. The data was corrected for acceptance, including reconstruction efficiency, and radiative effects, and binned in the following center-of-mass $(\mathrm{CM})$ variables: $p \pi^{+}$ 

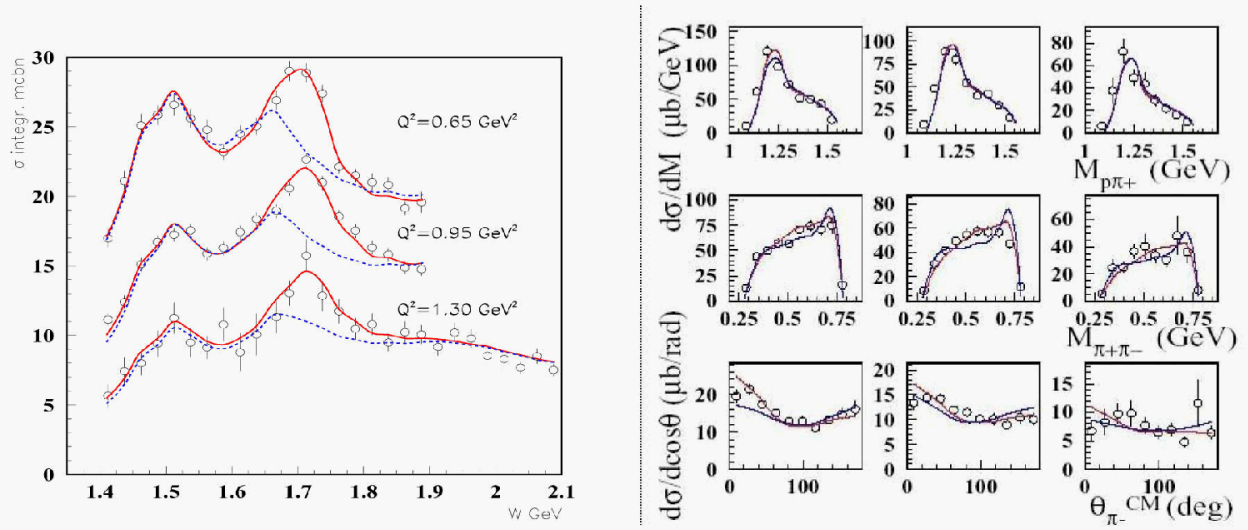

FIGURE 3. Left: Total cross section for $e p \rightarrow e^{\prime} p \pi^{+} \pi^{-}$for three different values of $Q^{2}$. Right: Differential cross sections from CLAS for the W bin $1.7-1.725 \mathrm{GeV} / \mathrm{c}^{2}$ for the three different $Q^{2}$ intervals. The blue line corresponds to a fit where the hadronic parameters and position of the $D_{13}(1700)$ were allowed to vary. The red line corresponds to a fit where the photocouplings of all three states: $D_{13}(1700), P_{13}(1720)$, and $P_{11}(1710)$ were allowed to vary, keeping the hadronic couplings fixed.

invariant mass, $\pi^{+} \pi^{-}$invariant mass, $\pi^{-}$solid angle, , and $y$, the angle between the $p \pi^{+}$ and the hadronic plane. A model $[5,6]$ was used to describe the reaction in the kinematic range of interest as a sum of amplitudes of all possible production mechanisms of $\Delta \pi$ and $\rho \pi$. All other production mechanisms of $p \pi^{+} \pi^{-}$were parameterized as phase space. A detailed treatment was developed for the non-resonant contributions to $\Delta \pi$ [5], while $\rho \pi$ non-resonant contributions were described through a diffractive ansatz. The model included twelve resonances listed in Table 1. The data was fit to this model in two steps, first fitting the non-resonant components keeping the resonant parameters within allowed values, and then fixing the non-resonant part and varying the resonant parameters. Generally the data fit the model very well, except for $W \sim 1700 \mathrm{MeV}$. Here various fits were performed, described in Fig. 3 and Ref. [4]. We conclude that phenomenological calculations using existing PDG parameters provide poor agreement with our data. The solution is either to dismiss previously established hadronic parameters for the $P_{13}(1720)$, or introduce a new state with $J^{P}=\frac{3}{2}^{+}$determined from the fit to the data, with a strong $\Delta \pi$ coupling, and small $\rho N$ coupling.

TABLE 1. Resonant states included in the fit of $e p \rightarrow$ $e^{\prime} p \pi^{+} \pi^{-}$.

\begin{tabular}{||c|c||c|c||c|c||}
\hline State & $J^{P}$ & State & $J^{P}$ & State & $J^{P} \|$ \\
\hline$P_{11}(1440)$ & $\frac{1}{2}^{+}$ & $D_{13}(1520)$ & $\frac{3}{2}^{-}$ & $S_{11}(1535)$ & $\frac{1}{2}^{-}$ \\
$S_{11}(1650)$ & $\frac{1}{2}^{-}$ & $D_{15}(1675)$ & $\frac{5}{2}^{-}$ & $F_{15}(1680)$ & $\frac{1}{5}^{+}$ \\
$P_{13}(1720)$ & $\frac{3}{2}^{+}$ & $D_{13}(1700)$ & $\frac{3}{2}^{-}$ & $S_{31}(1620)$ & $\frac{1}{2}^{-}$ \\
$D_{33}(1700)$ & $\frac{3}{2}^{-}$ & $F_{35}(1905)$ & $\frac{5}{2}^{+}$ & $F_{37}(1950)$ & $\frac{7}{2}^{+}$ \\
\hline
\end{tabular}

CLAS has also investigated this final state through photoproduction. In this case the tagged photon beam was induced from a $2.4 \mathrm{GeV}$ electron beam, which provided 
TABLE 2. Baryon waves included in the fit of $\gamma p \rightarrow$

\begin{tabular}{c|c|c}
$p \pi^{+} \pi^{-}$. & \\
\hline$J^{P}$ & $\mathbf{M}$ & Isobars \\
\hline$\frac{1}{2}^{+}$ & $\frac{1}{2}$ & $\Delta^{++} \pi^{-}, \Delta^{0} \pi+$ \\
$\frac{1}{2}^{-}$ & $\frac{1}{2}$ & $\Delta \pi, p \rho$ \\
$\frac{3}{2}^{+}$ & $\frac{1}{2}, \frac{3}{2}^{2}$ & $(\Delta \pi)_{l=1}, p \rho_{s=\frac{1}{2}},(p \rho)_{s=\frac{3}{2} ; l=1,3}, N^{*}(1440) \pi$ \\
\hline
\end{tabular}

photon energies between $480 \mathrm{MeV}$ to $2.28 \mathrm{GeV}$ [2]. The analysis performed was a full partial wave analysis in the isobar model, and is described in detail in [7]. The observed intensity distribution is described as a square of appropriately summed amplitudes.

$$
I(\tau)=\left.\left.\sum_{\alpha} \sum_{\beta}\right|^{\alpha} \psi_{\beta}(\tau)\right|^{2}
$$

$\tau$ represents the set of variables necessary to define the configuration of the final state, and $\alpha$ represents non-interfering terms in the expansion, for example, the spins of initial state or final state particles, and $\beta$ represents the partial wave decomposition. The processes considered in this case include s-channel baryon resonance production, with decay modes $\Delta \pi$ and $\rho p$, as well as t-channel $\rho$ production. Generally, the amplitudes ${ }^{\alpha} \psi_{\beta}(\tau)$ can be factored into the product of a production amplitude and a decay amplitude for the given partial wave:

$$
{ }^{\alpha} \psi_{\beta}(\tau)={ }^{\alpha} V_{\beta}^{\alpha} A_{\beta}(\tau)
$$

Given the above description of the intensity distribution and an ensemble of experimental events, a likelihood function for the given experimental data set can be formed:

$$
L=\left[\frac{\bar{n}^{n}}{n} e^{-\bar{n}}\right] \prod_{i}^{n}\left[\frac{I\left(\tau_{i}\right)}{\int I(\tau) \eta(\tau) d \tau}\right]
$$

In practice, the logarithm of the likelihood function is maximized by varying the production amplitudes. The integral in Eq. 3 is calculated by Monte Carlo methods, as the acceptance function $\eta(\tau)$ in general is not known in an analytic form. CLAS is simulated by a detailed GEANT model [1].

In the case of the reaction $\gamma p \rightarrow p \pi^{+} \pi^{-}$, separate, independent fits were done on the data in $20 \mathrm{MeV}$ bins of photon beam energy. The waves included in the fit are listed in table 2. In Fig. 4 a comparison between the fit results and various differential cross sections for a sample bin, $1.72<\mathrm{W}<1.75\left(\mathrm{GeV} / \mathrm{c}^{2}\right)$ is shown, and demonstrates that the fit provides a very good description of all of the relevant differential cross sections. Fig. 5 show the partial wave decomposition of the $J^{P}=\frac{5}{2}^{+}$wave. One can clearly see the $\Delta \pi$ decay mode of the $N^{*}(1680)$. Fig. 6 shows the $P_{13}$ and $P_{33}$ waves: the $N^{*}(1720)$ and $\Delta(1600)$ can be seen in the partial wave decomposition.

The PWA fit can then be integrated over the entire phase space to determine the acceptance corrected total cross section. Figure 7 shows the total cross section measured in CLAS and compared to the ABBHHM [11] and CEA [10] results, as well as the various physics components. 

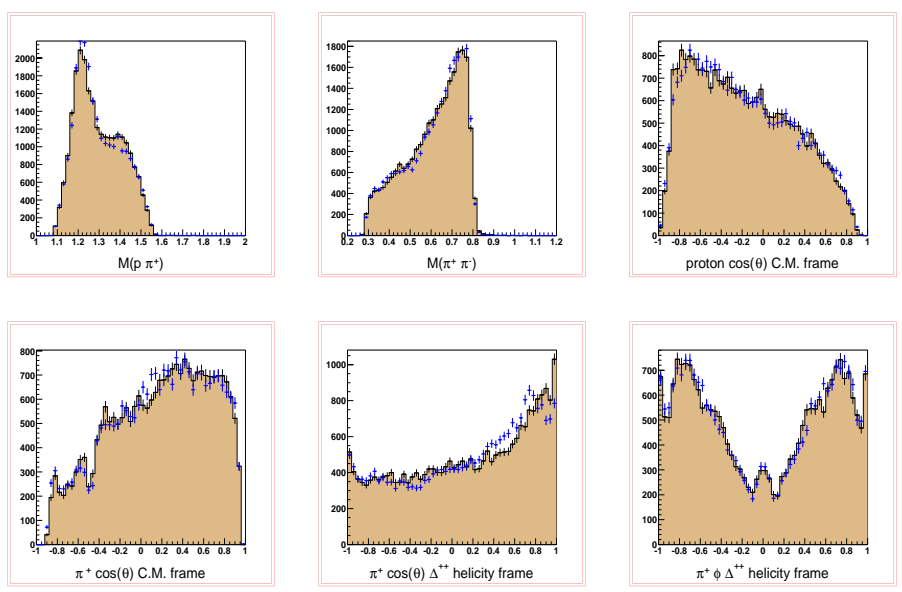

FIGURE 4. Comparison of the PWA fit to the data for the bin $1.72<\mathrm{W}<1.75\left(\mathrm{GeV} / \mathrm{c}^{2}\right)$ for the reaction $\gamma p \rightarrow p \pi^{+} \pi^{-}$The data is shaded, and the blue points are results of the fit.
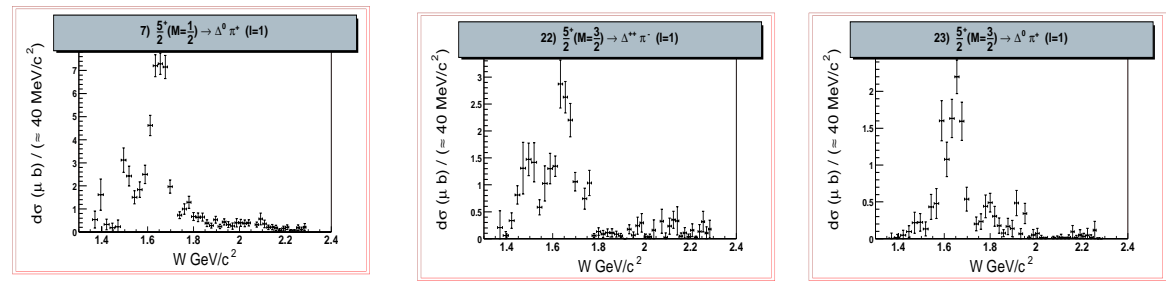

FIGURE 5. PWA results for the $J^{P}=\frac{5}{2}^{+}$waves in the reaction $\gamma p \rightarrow p \pi^{+} \pi^{-}$.

$$
\gamma P \rightarrow \pi \pi \pi N
$$

The CLAS collaboration has also studied the reactions $\gamma p \rightarrow \pi \pi \pi N$ at $5 \mathrm{GeV}$. Photoproduction is expected to be an excellent way to produce the lowest lying $J^{P C}=1^{-+}$exotic mesons. BNL has observed the $\pi_{1}(1600)$ decaying to the final state $\pi^{+} \pi^{-} \pi^{-}$[8][9].

In August and September in 2001 CLAS took data with a real photon beam with an energy range of $4.8 \mathrm{GeV}$ to $5.47 \mathrm{GeV}$. The $\gamma$ flux was $5 \times 10^{6} / \mathrm{sec}$ into an $18 \mathrm{~cm}$ liquid hydrogen target. The total raw sensitivity was 2.7 events/pb.

The partial-wave analysis of these data was performed using the same program developed for the baryon analysis, but in this case including various meson waves produced in the t-channel and decaying to $\pi \pi \pi$. Each event is considered in the framework of an isobar model: an initial decay of a parent particle into a $\pi \pi$ isobar and an unpaired pion followed by the subsequent decay of the isobar. Each partial wave is characterized by the quantum numbers $J^{P C}[$ isobar $] L M^{\varepsilon}$ - here $J^{P C}$ are spin, parity and C-parity of the partial wave; $M$ is the absolute value of the spin projection on the quantization axis; $L$ is the orbital angular momentum between the isobar and the unpaired pion. In this case 

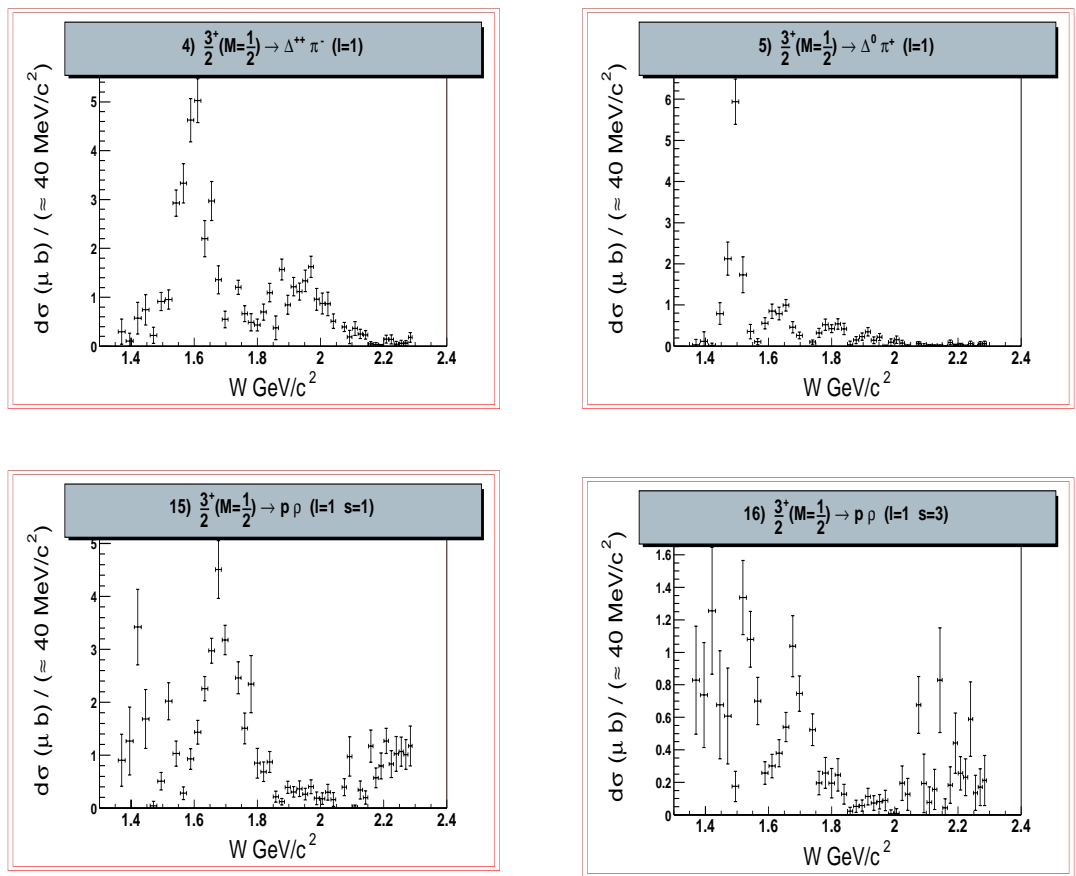

FIGURE 6. PWA results for the $J^{P}=\frac{3}{2}^{+}$waves in the reaction $\gamma p \rightarrow p \pi^{+} \pi^{-}$.
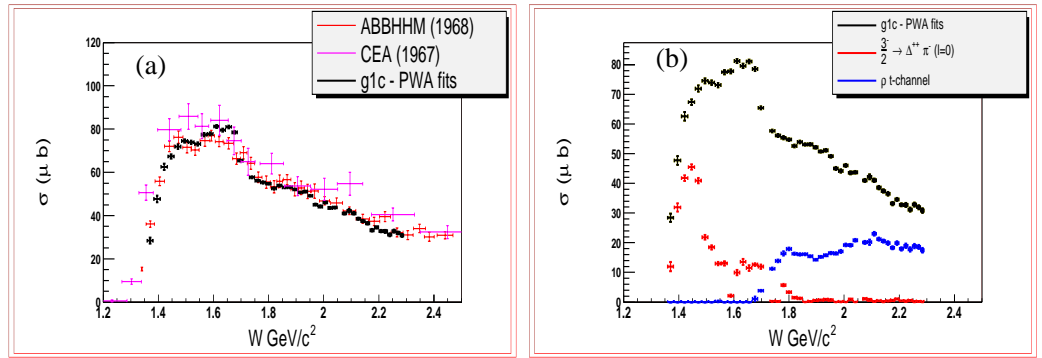

FIGURE 7. (a) Total cross section of the reaction $\gamma p \rightarrow \pi^{+} \pi^{-} p$ measured at CLAS, and compared to the ABBHHM and CEA results.(b) Various components of the total photon cross section from the PWA fits.

the spin-density matrix is expressed as eigenfunctions of the reflection operator through the reaction plane, $\varepsilon$ [12]. In the most general case, the rank of the spin-density matrix can be four, in practice fits were limited to rank one. Relativistic Breit-Wigner functions with standard Blatt-Weisskopf factors were used in the description of the $\rho(770)$, and $f_{2}(1270)$ isobars; the $\pi \pi \mathrm{S}$-wave, $\sigma$, was paramterized according to Au, Morgan, and Pennington [13]. 
For the reaction $\gamma p \rightarrow \pi^{+} \pi^{-} \pi^{0} p$ the three charged particles were measured in CLAS, while the $\pi^{0}$ was identified and measured from missing four-momentum. This reaction has considerable background from baryon resonances decaying to $p \pi$ through $\mathrm{t}$-channel exchange processes. For the partial wave analysis, this background was included as an interfering background, with the baryon resonance decay modeled as an S-wave decay. More sophisticated descriptions of the background did not significantly improve the results. A total of 28 meson waves and 21 background (baryon) waves were included, and are listed in Table 3. Many other waves were tried in the partial wave fits, and were determined to be very small. The resulting partial wave analysis of the $\pi \pi \pi$ for the isoscalar $1^{--}$, the exotic isovector $1^{-+}$, isovector $1^{++}$, and isovector $2^{++}$waves are shown in Fig. 8. There is clear evidence for the photoproduction of the $a_{1}(1260)$ as well as the $\omega(1650)$. There is some evidence for production of the $a_{2}(1320)$ as well. In addition, there is a strong signal that corresponds in mass and width to the exotic $\pi_{1}(1600)$.

TABLE 3. Waves included in the fit of $\gamma p \rightarrow \pi^{+} \pi^{-} \pi^{0} p$

\begin{tabular}{c|c|c|c}
\hline$J^{P C}$ & $M^{\varepsilon}$ & $\mathbf{L}$ & Isobars \\
\hline $1^{++}$ & $0^{+}, 1^{ \pm}$ & 0,2 & $\rho(770)$ \\
$1^{--}$ & $0^{-}, 1^{ \pm}$ & 1 & $\rho(770)$ \\
$1^{-+}$ & $0^{-}, 1^{+}$ & 1 & $\rho(770)$ \\
$2^{++}$ & $0^{-}, 1^{+}$ & 2 & $\rho(770)$ \\
$2^{-+}$ & $0^{+}, 1^{ \pm}$ & 0 & $f_{2}(1270)$ \\
$2^{-+}$ & $0^{+}$ & 2 & $f_{2}(1270)$ \\
$2^{-+}$ & $0^{+}, 1^{ \pm}$ & 1,3 & $\rho(770)$ \\
$2^{+-}$ & $0^{-}$ & 2 & $\rho(770)$ \\
$3^{--}$ & $0^{-}, 1^{+}$ & 3 & $\rho(770)$ \\
$3^{++}$ & $0^{-}, 1^{ \pm}$ & 1 & $f_{2}(1270)$ \\
$4^{++}$ & $0^{-}, 1^{-}$ & 3 & $f_{2}(1270)$ \\
\hline
\end{tabular}

From the same data run the reaction $\gamma p \rightarrow \pi^{+} \pi^{+} \pi^{-} n$ was studied. The three charged pions were measured in CLAS, while the neutron was identified and measured from missing four-momentum. Laboratory angle cuts on the pions, as well as selecting low $t$ events, greatly reduces the baryon resonance background. Fig. 9 shows various distributions of the data: the $t^{\prime}$ distribution, the missing neutron mass, and di-pion effective masses.

The PWA results are shown in Fig. 10; waves included in the fit are listed in Table 4. While these results are very preliminary, there is a very clear signal for the $a_{2}(1320)$. There is some evidence for photoproduction of the $a_{1}(1260)$ and also the $\pi_{2}(1670)$. There is some strength in the exotic $J^{P C}=1^{-+}$partial wave near $1600 \mathrm{MeV} / \mathrm{c}^{2}$, but it is not conclusive.

\section{$\Theta^{+}$}

The existence of an exotic $\mathrm{S}=+1$ baryon has been suggested by several recent experiments [14]. CLAS has investigated the reaction $\gamma d \rightarrow K^{+} K^{-} p(n)$ where the final state neutron is reconstructed from missing four-momentum. If the proton is a spectator in the 

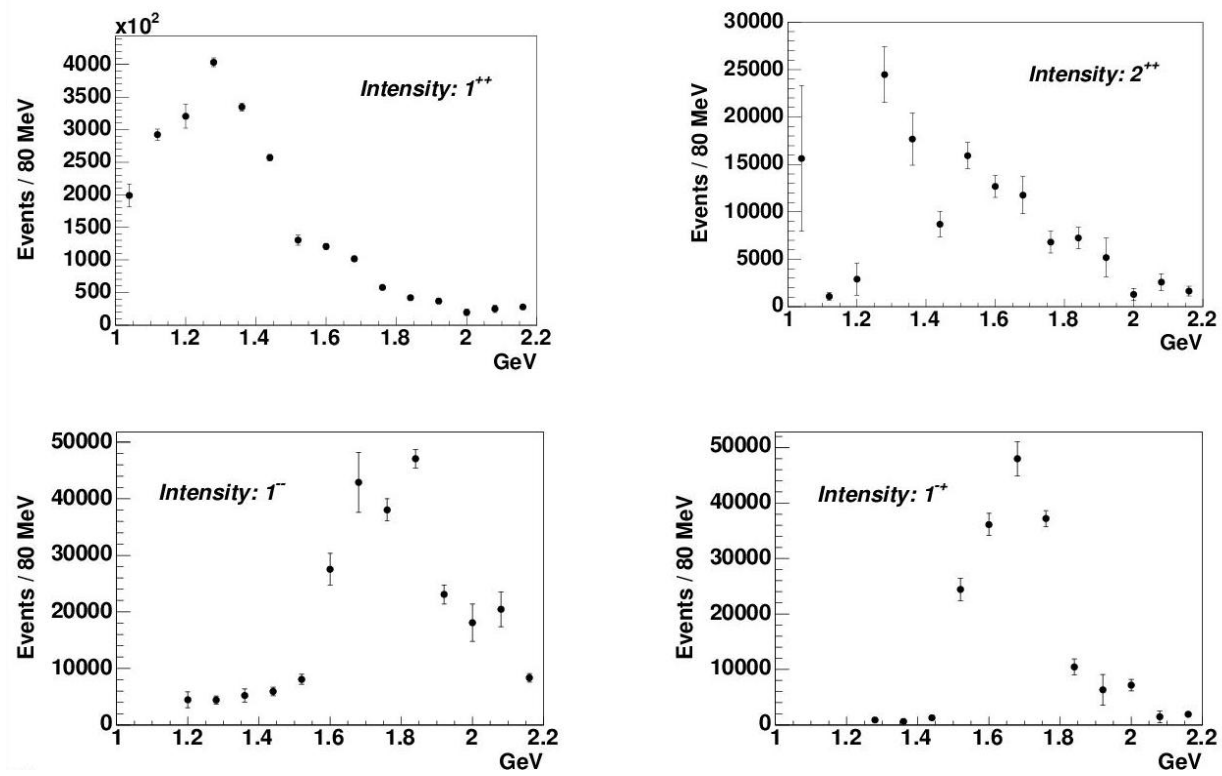

FIGURE 8. Partial wave decomposition of the reaction $\gamma p \rightarrow \pi^{+} \pi^{-} \pi^{0} p$ (a) $J^{P C}=1^{++}$isovector, (b) $J^{P C}=2^{++}$isovector (c) $J^{P C}=1^{--}$isoscalar (d) $J^{P C}=1^{-+}$isovector
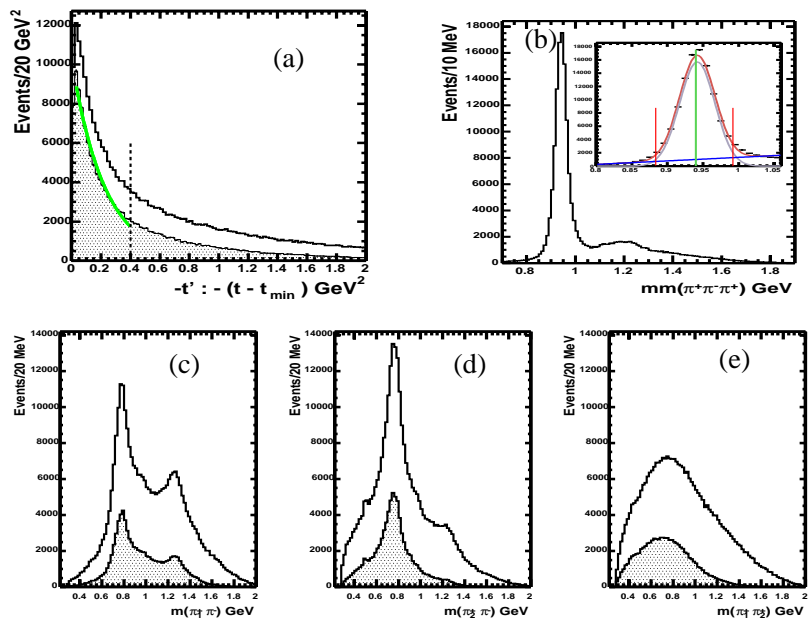

FIGURE 9. Various distributions of the reaction $\gamma p \rightarrow \pi^{+} \pi^{+} \pi^{-} n$ at $5.2 \mathrm{GeV}$. (a) $\mathrm{t}$ ' distribution. Events to the left of the dotted line were selected for the PWA. The shaded area has cuts on the pion laboratory angles described in the text. (b) Missing mass off of $\pi^{+} \pi^{+} \pi^{-}$showing the missing neutron. (c) Mass of the $\pi^{-}$and slow $\pi^{+}$showing both the $\rho$ and $f_{2}(1270)$ isobars. (d) Mass of the $\pi^{-}$and fast $\pi^{+}$. (e) Mass of $\pi^{+} \pi^{+}$. (c),(d), and (e) shaded areas are events selected for the final partial wave analysis. 
TABLE 4. Partial waves included in the fit of $\gamma p \rightarrow$

\begin{tabular}{c|l|c|c|c}
\hline$J^{P C}$ & $m^{\varepsilon}$ & L & Isobar & \# Waves \\
\hline $0^{-+}$ & $0^{+}$ & 0 & $\sigma$ & 1 \\
\hline $0^{-+}$ & $0^{+}$ & 1 & $\rho(770)$ & 1 \\
\hline $1^{++}$ & $0^{+}, 1^{ \pm}$ & 0,2 & $\rho(770)$ & 6 \\
\hline $1^{++}$ & $0^{+}, 1^{ \pm}$ & 1 & $\sigma$ & 3 \\
\hline $1^{-+}$ & $0^{-}, 1^{ \pm}$ & 1 & $\rho(770)$ & 3 \\
\hline $2^{++}$ & $0^{-}, 1^{ \pm}, 2^{ \pm}$ & 2 & $\rho(770)$ & 5 \\
\hline $2^{-+}$ & $0^{+}, 1^{ \pm}$ & 1,3 & $\rho(770)$ & 6 \\
\hline $2^{-+}$ & $0^{+}, 1^{ \pm}$ & 2 & $\sigma$ & 3 \\
\hline $2^{-+}$ & $0^{+}, 1^{ \pm}$ & 0,2 & $f_{2}(1270)$ & 6 \\
\hline Background & & & &
\end{tabular}
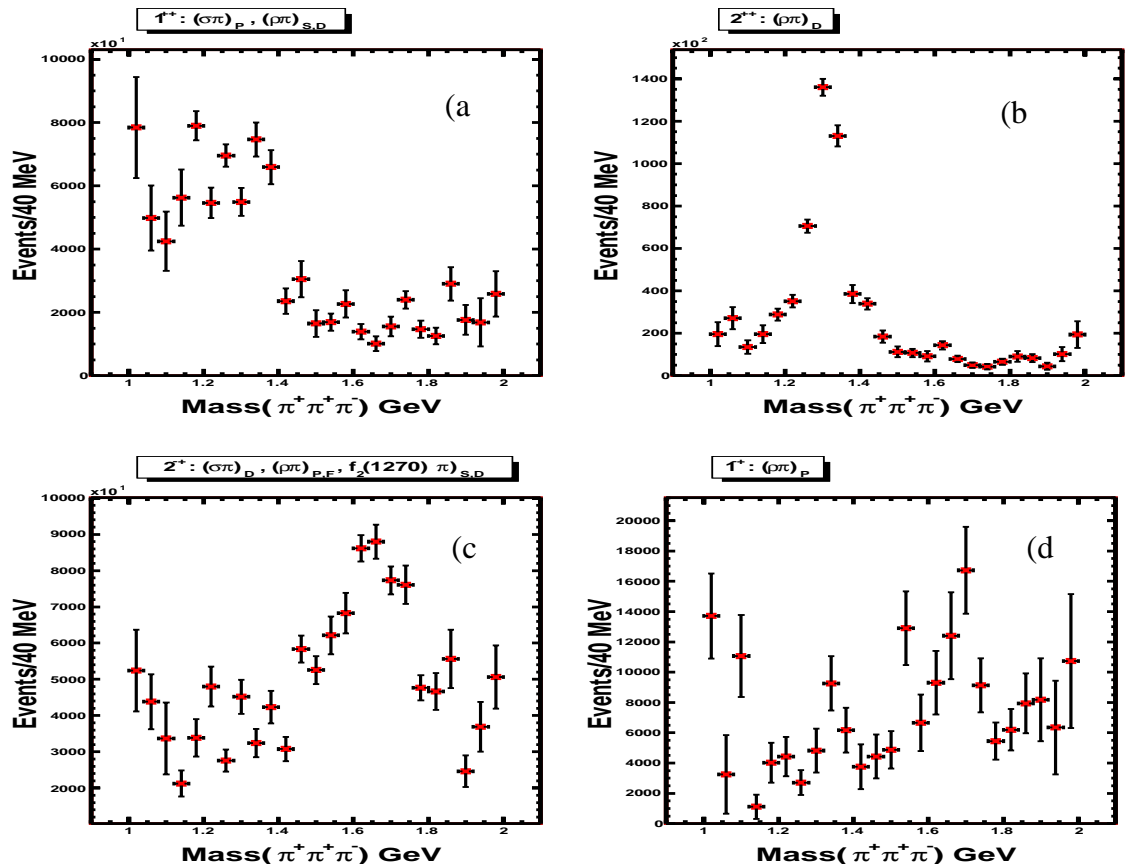

FIGURE 10. Partial wave decomposition of the reaction $\gamma p \rightarrow \pi^{+} \pi^{+} \pi^{-} n$. (a) $J^{P C}=1^{++}$partial wave. (b) $2^{++}$partial wave. (c) $2^{-+}$wave. (d) Exotic $1^{-+}$wave. 


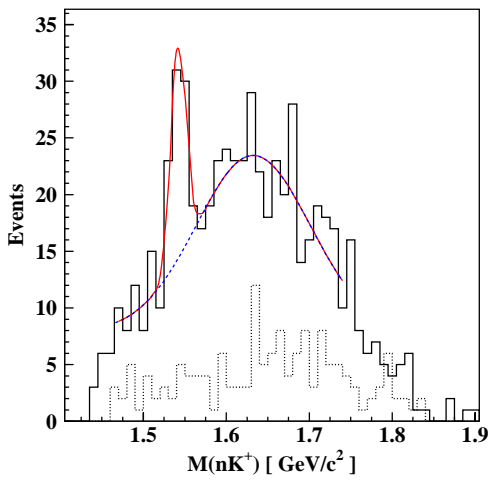

FIGURE 11. Invariant mass of the $n K^{+}$showing a sharp peak at $1.542 \mathrm{GeV} / \mathrm{c}^{2}$. A fit (solid line) to the peak with a smooth background (dashed line) gives a statistical significance of $5.8 \sigma$.

interaction, its Fermi momentum is not sufficient to have it detected in CLAS. In some fraction of the events, however, both the neutron and proton partake in the interaction, perhaps through rescattering. The data presented here were taken with the CLAS tagged photon beam, with the incident electron at 2.474 and $3.115 \mathrm{GeV}$. The tagged photon flux was approximately $4 \times 10^{6} \gamma$ per second. The integrated tagged photon flux above 1.51 $\mathrm{GeV}$ was $2.34 \times 10^{12}$. The beam was incident on a $10 \mathrm{~cm}$ liquid deuterium target.

Events were selected with the neutron momentum above $80 \mathrm{MeV} / \mathrm{c}$. Known resonances, the $\phi(1020)$ and $\Lambda(1520)$ were removed from the analysis. Monte carlo simulations indicated that for $\Theta^{+}$events the $K^{+}$momentum rarely exceeded $1.0 \mathrm{GeV} / \mathrm{c}$, and events with $p_{K^{+}}>1.0 \mathrm{GeV} / \mathrm{c}$ were removed. The resulting invariant mass of the neutron and $K^{+}$is shown in Fig. 11, and shows a 5.8 standard deviation peak over a smooth background at a mass of $1.542 \mathrm{GeV} / \mathrm{c}^{2}$.

The CLAS collaboration has also searched for the $\Theta^{+}$baryon in $\gamma p$ interactions. In three separate data runs, with slightly different running conditions, CLAS took data with an $18 \mathrm{~cm}$ liquid hydrogen target and photon energies greater than $3.2 \mathrm{GeV}$. Two data runs (labeled a and b) had identical geometrical acceptance and trigger conditions, but different beam energies. Run $a$ had a photon beam energy range 3.2-3.95 GeV, run $b 3-5.25 \mathrm{GeV}$, and run $c 4.8-5.47 \mathrm{GeV}$. Run $c$ triggered on the events with at least 2 out of 6 CLAS sectors having signals, while runs $a$ and $b$ triggered on events with hits in opposite sectors. Runs $a$ and $b$ have the hydrogen target in the standard position, but in run $c$ the target was moved upstream by 1 meter to improve CLAS acceptance in the forward direction. The estimated combined $a$ and $b$ integrated luminosity is about 2 events/pb, and run $c$ has about 2.7 events/pb.

Events having a $\pi^{+}, K^{+}$, and $K^{-}$in the final state were selected for the analysis of the reaction $\gamma p \rightarrow \pi^{+} K^{-} K^{+} n$. The missing mass distribution off of the $\pi^{+} K^{+} K^{-}$shows a very clear neutron peak in each data set; these events were selected for further analysis. 

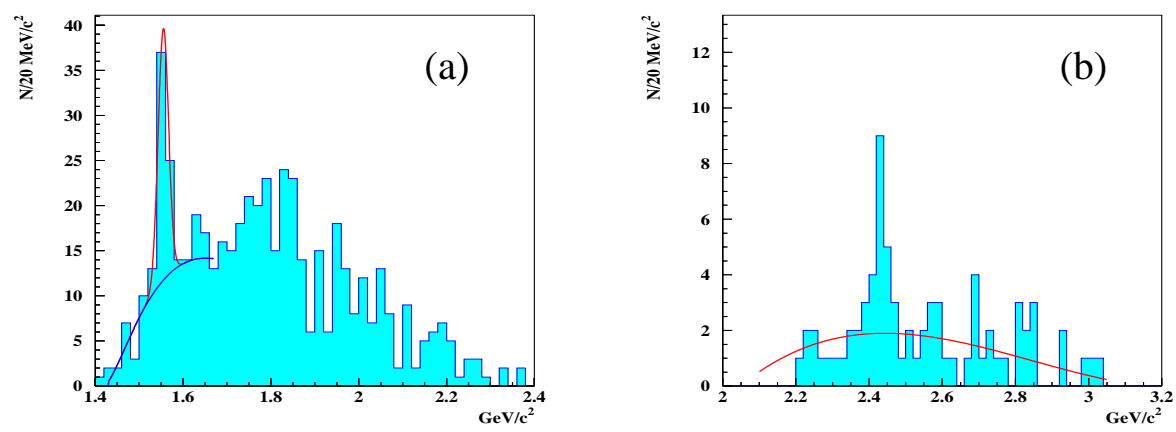

FIGURE 12. (a) The $M_{n K^{+}}$invariant mass spectrum in the reaction $\gamma p \rightarrow \pi^{+} K^{-} K^{+}(n)$ with the cut $\cos \theta_{\pi^{+}}^{*}>0.8$ and $\cos \theta_{K^{+}}^{*}<0.6 . \theta_{\pi^{+}}^{*}$ and $\theta_{K^{+}}^{*}$ are angles between the $\pi^{+}$and $K^{+}$mesons and photon beam in the center of mass system. The background function we used in the fitting was obtained from the phase space simulation we completed. (b) The $M_{n K^{+} K^{-}}$invariant mass spectrum calculated from the missing mass off of the $\pi^{+}$in the reaction $\gamma p \rightarrow \pi^{+} K^{-} K^{+}(n)$ with the cuts $\cos \theta_{\pi^{+}}^{*}>0.8$ and $\cos \theta_{K^{+}}^{*}<0.6$. $\theta_{\pi^{+}}^{*}$ and $\theta_{K^{+}}^{*}$ are angles between the $\pi^{+}$and $K^{+}$mesons and photon beam in the center of mass system. The events in this plot have $M\left(K^{+} n\right)$ between 1.54 and $1.58 \mathrm{GeV} / \mathrm{c}^{2}$. The shape of the background curve was obtained from our phase space simulation.

$\phi(1020)$ events were removed from the data sample by cutting events with $K^{+} K^{-}$ effective mass less than $1.06 \mathrm{GeV} / \mathrm{c}^{2}$.

Two angular cuts in the center of mass system were applied to extract the signal and suppress the background. Structure in the region of $1.55 \mathrm{GeV} / \mathrm{c}^{2}$ appears after cutting $\cos \theta_{\pi^{+}}^{*}>0.8$, where $\theta_{\pi^{+}}^{*}$ is the center-of-mass angle between $\pi^{+}$and the photon beam, and corresponds roughly to $-t<0.28 \mathrm{GeV} / \mathrm{c}^{2}$ in our beam energy range, where $t=(k-p)^{2}, k$ is the photon 4-momentum, and $p$ is the pion 4-momentum. The reaction $\gamma p \rightarrow \pi^{+} K^{-} K^{+} n$ is dominated by meson resonance production decaying to $K^{+} K^{-} \pi^{+}$ and small momentum transfer to the proton, and the excitation of baryon resonances decaying to $n \pi^{+}$. These processes have the $K^{+}$moving forward in the center of mass system. To suppress such backgrounds, a cut was applied to select events having a positive kaon going in the backward direction with $\cos \theta_{K}^{*}<0.6$. The $\theta^{+}$peak was clearly observed in each of the three data sets; the resulting $n K^{+}$mass spectrum were combined and are shown in Fig. 12(a).

The $n K^{+}$effective mass distribution was fitted by the sum of a Gaussian function and a background function obtained from phase space simulation. The fit parameters are: $N_{\text {events }}=41 \pm 10, M=1555 \pm 1 \mathrm{MeV} / \mathrm{c}^{2}, \sigma=11 \pm 3 \mathrm{MeV} / \mathrm{c}^{2}$. The mass scale uncertainty is estimated to be $\pm 10 \mathrm{MeV} / \mathrm{c}^{2}$. This uncertainty is mainly due to the momentum calibration of the CLAS detector and the photon beam energy calibration. The statistical significance for the fit in Fig. 12 calculated over a $40 \mathrm{MeV} / \mathrm{c}^{2}$ mass window is $7.8 \sigma$. The fact that the angular cuts we applied enhanced the $\Theta^{+}$signal suggests the possible production of a $N^{*} / \Delta^{*}$ that decays to $\Theta^{+}$and $K^{-}$; if the $\Theta^{+}$is an isoscalar, only 
an intermediate $N^{*}$ ia possible. For the events with $n K^{+}$effective mass between 1.54 and $1.58 \mathrm{GeV} / \mathrm{c}^{2}$, the missing mass off of the $\pi^{+}$is shown in Fig. 12(b).

\section{SUMMARY}

CLAS at Jefferson Laboratory has a continuing rich program in hadron spectroscopy. New high-quality data from the electroproduction and photoproduction of multi-meson decays of baryon resonances, eg. $p \pi^{+} \pi^{-}$is currently being analyzed. Such studies will shed light on the missing baryon question. In addition, CLAS is currently analyzing data from the photoproduction of mesons. Initial partial wave analysis results are now becoming available. New proposals for high statistics real photon beam experiments, as well as near-real photons using small angle electron scattering, which produces linearly polarized photons, are in preparation. In addition, CLAS, in $\gamma d$ and $\gamma p$ interactions, has contributed significantly to the rapidly expanding study of exotic penta-quark states such as the $\Theta^{+}(1540)$.

\section{ACKNOWLEDGMENTS}

I wish to thank all of my collaborators at CLAS for their help with this talk, as well as the extraordinary efforts of the CEBAF staff which have made this program possible. This work is supported by the Department of Energy under contract DE-AC05-84ER40150.

\section{REFERENCES}

1. B. A. Mecking, et al. Nucl. Instrum. Meth. A503,513-553 (2003).

2. D. I. Sober, et al. Nucl. Instrum. Meth. A440, 263 (2000).

3. M. D. Mestayer, et al. Nucl. Instrum. Meth. A449, 81 (2000).

4. $\quad$ M. Ripani, et al. Phys. Rev. Lett. 91, No. 2:022002-1 (2003).

5. M. Ripani, et al. Nucl. Phys. A672, 220 (2000).

6. V. Mokeev, et al. Phys. At. Nucl 64, 1292 (2001).

7. John P. Cummings and Dennis P. Weygand, "An Object-Oriented Approach to Partial Wave Analysis", arXiv:physics/030952 (submitted to Nucl. Instrum. Meth) (2003).

8. $\quad$ G. S. Adams, et al. Phys. Rev. Lett. 81:5760-5763 (1998).

9. $\quad$ S. U. Chung, et al. Phys. Rev. D65:072001 (2002).

10. H. R. Crouch, Jr., et al., Phys. Rev.145, 994-1000 (1966).

11. ABBHHM Collaboration, Phys. Rev. Lett. 175, 1669 (1968).

12. S. U. Chung and T. L. Trueman, Phys. Rev. D11, 633

13. K. L. Au, D. Morgan, and M. R. Pennington, Phys. Rev. D35, 1633 (1987).

14. T. Nakano, et al., Phys. Rev. Lett. 91, 012002 (2003).

15. V. Barmin, et al. Phys. At. Nucl 66:1715-1718 (2003). 